\title{
Problemática fiscal de las finanzas territoriales en el Distrito de Barranquilla durante la vigencia del año 2015 al $2017^{1}$
}

\section{Problem fiscal of regional finances in the district of Barranquilla during the term of 2015 to 2017}

\author{
Karen Donado Consuegra ${ }^{2}$ \\ Natali Corcho Urruchurto ${ }^{3}$ \\ Yudis Molina Gómez ${ }^{4}$ \\ RESUMEN
}

El presente artículo establece una visión especial de la problemática fiscal de las finanzas territoriales en el Distrito Barranquilla, durante la vigencia del año 2015 al 2017; ya que en la actualidad existen factores que limitan la autonomía administrativa y presupuestal de las entidades territoriales del país, como es el déficit económico actual como resultado de una política fiscal que cada día se torna más insostenible y qué es en gran parte debido al manejo indebido de las finanzas públicas territoriales, al no distribuir de forma correcta los recursos disponibles se genera una deuda con acreedores y entidades financieras. Uno de los factores que más incide es la falta de "Cultura Tributaria", situación relevante que ha sido tomada como justificación a la promulgación de diversas y constantes reformas tributarias que han generado regulaciones aisladas en materia tributaria.

\section{Palabras claves:}

Problemática Fiscal, Finanzas, Descentralización Territorial, Distrito de Barranquilla, Recursos Propios.

\footnotetext{
${ }^{1}$ Este artículo es el resultado de una investigación en materia de problemática fiscal de las finanzas Territoriales en el Distrito De Barranquilla durante la vigencia del año 2015 Al 2017 realizado en el marco del módulo en entidades territoriales de la Especialización de Derecho Administrativo de la Universidad Simón Bolívar de Barranquilla.

${ }^{2}$ Abogada, estudiante de II Semestre de Especialización de Derecho Administrativo de la Universidad Simón Bolívar. Email: Karend28@hotmail.

${ }^{3}$ Abogada, estudiante de II Semestre de Especialización de Derecho Administrativo de la Universidad Simón Bolívar. Email:natalicorcho@gmail.com

${ }^{4}$ Abogada, estudiante de II Semestre de Especialización de Derecho Administrativo de la Universidad Simón Bolívar. Email:yudismog@hotmail.com

ISSN: 2346-2787

Recibido: 1 de Mayo de 2018

Aprobado: Junio 29 de 2018.
} 


\begin{abstract}
This article establishes a special vision of the problematic tax of regional finances in the District of Barranquilla, during the term of 2015 to 2017, because at the present time there are factors that limit the administrative autonomy and budget of the territorial entities of the country, as is the deficit of current economic as a result of a fiscal policy that every day becomes more untenable, and what is in large part due to the improper management of public finances territorial, by not distributing properly the available resources generated a debt with creditors and financial institutions. One of the factors that most affects it is the lack of "Tax Culture", situation relevant that has been taken as a justification for the enactment of diverse and constant tax reforms that have generated regulations isolated in tax matters.
\end{abstract}

\title{
Key words:
}

Problems fiscal, Financial, Territorial Decentralization, District of Barranquilla, Own resources.

\section{Introducción}

El sistema económico de Colombia, viene padeciendo las consecuencias del manejo indebido de las finanzas públicas, hecho que ha generado un aumento del déficit fiscal en el país ya que al no distribuir de forma correcta los recursos disponibles se genera una deuda con acreedores y entidades financieras.

Salomón Kalmanovitz, le atribuye estos sucesos a qué históricamente Colombia proviene de una tradición centralista y de unidad de Iglesia y Estado. Sosteniendo su tesis en lo siguiente:

"El Centralismo implicaba una tributación sin representación la cual era evadida por los ciudadanos a manera de resistencia (...)". Además de lo anterior, alega lo siguiente: "La segunda característica implicaba la ausencia de libertad de conciencia, y por lo tanto, de expresión lo que permitía mostrar al Estado como una figura intocable y milagrosa. Asegura además que desde la colonia el país se ha acostumbrado a qué el 
Gobierno se apropia para sí, de todas las oportunidades de negocio" (Kalmanovitz, 1989).

Hoy por hoy, nuestro país carece de un estatuto tributario territorial, lo cual ha generado un impacto negativo en las finanzas públicas, desencadenando una libertad sin límites que le ha permitido a las entidades territoriales, realizar manejos incorrectos de los recursos públicos sin temor de asumir responsabilidad alguna. Muestra de ello, está en la obsoleta legislación que excluye conceptos relacionados con la actual tributación territorial y las posibilidades de gestión de los recursos que integran los activos del patrimonio público, siendo este último el mayor problema de las entidades territoriales al no distribuir adecuadamente sus recursos, pero además, que el modelo de descentralización económica no cuente con una vigilancia y control fuerte y eficaz, permitiendo que esta situación siga contribuyendo al aumento de la deuda pública de manera insostenible.

Tal es el caso de la ciudad de Barranquilla que ha estado, enmarcada en un crecimiento desbordado de los gastos con crecimiento leve de los ingresos, que se traduce en un presupuesto de gastos comprometido en su totalidad, "de modo que los recursos propios no tienen el mismo comportamiento, situación que conduce a un endeudamiento con acreedores y entidades financieras" (Villanueva, 2011).

Logrando así lo que se conoce como un déficit fiscal primario, donde los ingresos no alcanzan para pagar los intereses que se originan por los compromisos financieros, lo cual genera alto endeudamiento; a su vez se sacrifican las necesidades más sentidas de la comunidad, por el endeudamiento fiscal.

Un mejor desempeño fiscal se asocia a realizar un seguimiento financiero de las entidades territoriales como la autofinanciación de sus gastos de funcionamiento, el porcentaje de recursos destinados a pagar las deudas, la dependencia de las 
transferencias que se realizan y de las regalías, generación de recursos propios, porcentajes de recursos que están destinado a la inversión, mayores niveles de desarrollo territorial, lo cual se traduce en un aumento del valor agregado municipal, mayor provisión de bienes y servicios públicos y una disminución en la tasa de desempleo de la comunidad.

Si una entidad territorial llámese Municipio, Departamento o en este caso Distrito tiene un indicador positivo relacionado con los recursos que administra, dará una mayor posibilidad de satisfacer necesidades que en realidad son insatisfechas a través de la acción pública. Tener un indicador positivo debe significar mayores inversiones en obras físicas necesarias como colegios, hospitales, vías, bibliotecas, etc.; pero también debe realizar una inversión de carácter social como la seguridad alimentaria y nutricional, programas para acceder a sistemas educativos mejorando su calidad, programas para prevenir la violencia y una sana convivencia entre otros.

En este sentido se estructura la siguiente pregunta: ¿Cuáles son las principales causas de la problemática fiscal de las finanzas territoriales en el Distrito de Barranquilla durante la vigencia de 2015 a $2017 ?$

Se pretende a través de este artículo, elaborar un examen crítico de la institucionalidad que gobierna la descentralización fiscal y administrativa en Colombia, como Estado social de Derecho; exponiendo las principales dificultades que afronta; $y$, formular recomendaciones con miras a que se gestionen de mejor manera los recursos en el Distrito de Barranquilla, con miras a su propio desarrollo.

\section{Metodología}

Para el desarrollo de este artículo se utilizó una metodología que responde al tipo de investigación jurídica básica o pura, desde el modelo cualitativo- descriptivo, en el marco de la problemática fiscal del distrito, utilizando como población al Distrito de 
Barranquilla, a través del análisis y revisión documental de información referente al manejo de las finanzas territoriales; desde un enfoque crítico y analítico.

\section{Descentralización Fiscal y Sus Debilidades Institucionales.}

La Descentralización Fiscal, obedece a una forma de gobierno en el cual el nivel central delega competencias fiscales hacia los entes territoriales a manera de transferencia de recursos denominados ingresos, para que estos los administren adecuadamente y financien sus gastos públicos. Lo anterior, con el objeto de entregar un recurso a la comunidad que integra a cada ente territorial y que esta reciba a su vez un beneficio social, generando así, un crecimiento económico en cada territorio.

Siguiendo este hilo conductor, Gerardo A. Villa D. en su ensayo sobre Economía Regional titulado "Colombia: Gasto Público Social Regional 1997 -2003 Con Breves Comparaciones Internacionales", manifestó lo siguiente:

"En términos prácticos la descentralización hace parte de una estrategia para la orientación y movilización de recursos en tal forma que se obtenga un beneficio neto al momento de entregar servicios sociales a una comunidad." (Villa, 2005)

La Descentralización Fiscal en Colombia, desde la constituyente de 1991 adoptó un modelo de mayor apertura económica, sin embargo, ante la ausencia de una normatividad verdaderamente severa, la política fiscal de este país se ha tornado cada vez más insostenible afectando principalmente a las clases bajas y medias, pues las reformas tributarias sancionadas lejos de fortalecer el régimen tributario lo que ha generado es un aislamiento a todos esos derechos de fundamentales económicos, sociales y culturales proclamados en la Constitución Política de 1991, al punto que "las reformas económicas han buscado crear un Estado austero, cuya política presupuestal evite el excesivo endeudamiento, como condición necesaria de la estabilidad macroeconómica" (Hernández Gamarra \& Barreto Nieto, 2018), afectando a las clases menos favorecidas. 
Así mismo, con la Constitución Política de 1991 y sus normas reglamentarias, se fijaron nuevos criterios normativos sobre el reparto y usos de los recursos fiscales a nivel territorial por parte de la Nación hacia los diferentes Departamentos, Municipios y/o Distritos Especiales, estableciendo una destinación prioritaria de estos recursos para educación y salud, autorizando al gobierno a través de los artículo 356 y 357 a expedir la Ley 60 de 1993 con la que inicialmente se reglamentó la distribución de los recursos fiscales a los diferentes entes territoriales Posterior a lo anterior y mediante la reforma constitucional de 2001 en el Acto Legislativo 01 de 2001 y con el propósito de detener la problemática fiscal en las finanzas del gobierno central por el aumento de transferencia territoriales, se creó el Sistema General de Participaciones (SGP).

Además de lo anterior, nuestra Carta Magna de 1991, en su artículo 332 estableció "EI Estado es propietario del subsuelo y de los recursos naturales no renovables, sin perjuicio de los derechos adquiridos y perfeccionados con arreglo a las leyes preexistentes. (Constitución Política , 1991 ), dando paso al Régimen de Regalías como un Sistema General, producto del pago realizado por compañías petroleras y mineras al Estado Colombiano por la explotación de estos recursos naturales. En virtud a lo anterior y a lo contemplado por los artículo 360 y 361 del mismo extracto normativo, se dispuso que todo ingreso nacional proveniente de las regalías, conforman un Fondo Nacional disponible a ser descentralizado territorialmente conforme a los parámetros de ley fijados para su distribución y destinación. No obstante, con el paso del tiempo y pese a las diversas reformas sancionadas a la fecha, se siguen manifestando innumerables irregularidades tanto en la distribución de recursos como en la asignación de regalías a nivel territorial, evidenciando así las debilidades institucionales persistentes de ambos sistemas (SGP y SGR).

De igual forma, para el nivel territorial se expidieron normas que han tenido como propósito garantizar la disciplina fiscal de las entidades territoriales, limitando así su autonomía para generar recursos fiscales, ya que la determinación de tributos territoriales es competencia exclusiva del Congreso de la República. De lo anterior y tomando como referencia el contexto de las entidades territoriales, es válido deducir 
que el gobierno nacional ha liderado las reformas de tipo normativo desde un enfoque propio.

Lo anterior a trayendo consigo falencias del Estado frente al proceso de descentralización, de acuerdo a los postulados de Maldonado cuatro principales falencias y carencia:

a) Insuficiencia de políticas públicas que retomen el tema de la descentralización desde una discusión amplia que permita en su construcción los valores democráticos; b) carencia de articulación y coordinación entre el poder central y las entidades territoriales; c) ausencia en los sistemas de información y evaluación de los efectos de los procesos adelantados de la descentralización, impidiendo un análisis que permita detallar los avances y retrocesos del modelo; y d) un reducido apoyo técnico y administrativo desde el nivel central a los municipios más pequeños del país, los cuales han carecido de formulaciones pertinentes frente a su propio desarrollo local y los principios del Estado. (Maldonado, 2010)

Todas estas razones expuestas, sumadas al manejo indebido de los recursos públicos a nivel nacional y territorial, a la ausencia de equidad e igualdad al momento de distribuir la transferencia de recursos, lo que han logrado es disminuir los ingresos de la Nación y aumentar el déficit fiscal.

\section{Organización Territorial de Ingresos Fiscales.}

A través de los ingresos a las Entidades Territoriales se logra dar cumplimiento a sus objetivos y funciones especialmente por los bienes y servicios públicos.

Estos ingresos territoriales no son más que las rentas que los Departamentos, Municipios y Distritos y sus Entidades Descentralizadas reciben para dar cumplimiento a sus objetivos establecidos legal y constitucionalmente, en el caso del Distrito de Barranquilla establecidos por el Concejo dentro de los límites que ordena la Ley. 
En un comienzo los ingresos fiscales se recopilaban de forma voluntaria entregada por los asociados pero cuando se comenzaron a estructurar los Estados y en este sentido su descentralización se expidieron leyes que hicieron de carácter obligatorio que los ciudadanos entregaran dichas contribuciones pecuniarias.

En Colombia dichos ingresos fiscales se clasifican en ordinarios y extraordinarios. Los ordinarios son aquellos que se recopilan de forma constante y los extraordinarios no son recurrentes.

Según el Estatuto Orgánico del Presupuesto Decreto 111 de 1996 los entes territoriales clasifican sus ingresos en corrientes, fondos especiales, contribuciones parafiscales, recursos de capital e ingresos de los establecimientos públicos.

Gracias a estos recaudos cuentan con el derecho a establecer aquellos tributos que consideren necesarios para dar cumplimiento a sus funciones y poder ser partícipes de las rentas de carácter nacional y conservar la estructura establecida por las rentas nacionales.

\section{Descentralización en el Distrito de Barranquilla.}

La Constitución Política de Colombia en su artículo 328 establece un régimen de carácter específico para los municipios que poseen características especiales y por ello pasan a ser distritos. Barranquilla se encuentra dentro de esta calificación ya que en 1993 mediante un acto legislativo adquirió esta connotación acompañada de todas las responsabilidades y beneficios que esto traía.

A partir del año 2002 se expide la Ley 768 a fin de establecer un régimen para los distritos y la cual tiene como objeto "dotar a los distritos de Barranquilla, Santa Marta y Cartagena de Indias de las facultades, instrumentos y recursos que les permitan cumplir las funciones y prestar los servicios a su cargo; promover el desarrollo integral 
de su territorio para contribuir al mejoramiento de la calidad de vida de sus habitantes, a partir del aprovechamiento de sus recursos y ventajas derivadas de las características, condiciones y circunstancias especiales que prestan éstos, considerados en particular".

Una vez promulgada esta Ley se inició el proceso de ordenamiento territorial por localidades. De esta manera nace "el estatuto orgánico de las localidades en Barranquilla" a través del Acuerdo 017 de 2002, el cual posteriormente fue modificado a través del Acuerdo Distrital 006 de 2006, el cual llega a formalizar el proceso de descentralización interterritorial del distrito de Barranquilla.

Según el Acuerdo Distrital 006 de 2006 en su Título I Localidades de Barranquilla, el Distrito Especial, Industrial y Portuario de Barranquilla se organiza en 5 localidades de la siguiente manera: Localidad de Murillo Sur Occidente, Localidad de Murillo Sur Oriente, Localidad Norte - Centro Histórico, Localidad Metropolitana y Localidad Riomar.

Posterior a esto se promulga la Ley 1617 de 2013 la cual contiene las disposiciones que conforman el Estatuto Político, Administrativo y Fiscal de los Distritos y por la cual se expide el nuevo régimen para los Distritos especiales es decir la nueva Ley de Distritos.

Recursos Propios Del Distrito De Barranquilla Y Su Enfoque Para La Ejecución Del Plan De Desarrollo 2016 - 2019 "Barranquilla Capital De Vida"

El Plan Nacional de Desarrollo en Colombia, es un documento legal que contiene todos los objetivos y lineamientos de gobierno, trazados por cada Presidente de la República, al igual que las estrategias a implementar por parte de este y su equipo de gobierno, para la ejecución del mismo durante su período de mandato.

La Constitución Política de 1991 determina en su artículo 339, que el Plan Nacional de Desarrollo de cada gobierno colombiano, debe contener una parte general y una parte de inversión de las entidades públicas, orientadas al desarrollo económico, social y cultural a nivel nacional a través de sus entes territoriales, especificando las inversiones 
a realizar con recursos propios y las que serán con otros recursos financieros externos, determinando la sostenibilidad de estas inversiones, las facultades de comprometer vigencias futuras y el beneficio social de las mismas.

La ley orgánica 152 de 1994 contiene el marco legal del Plan Nacional de Desarrollo y en ella se establecen los principios generales de planeación, se designa la participación de las autoridades nacionales competentes para la elaboración del mismo y los lineamientos legales para su aprobación, socialización, ejecución y evaluación de resultados, señalando como responsable directo al Departamento Nacional de Planeación.

Por su parte, el Distrito Especial, Industrial y Portuario de Barranquilla, mediante Aprobación del Acuerdo Distrital No. 0011 de 2016 y conforme a lo dispuesto en el Art. 76 de la Ley 136 de 1994, adoptó en su actual gobierno su plan de desarrollo económico, social, ambiental y de obras públicas a nivel distrital (2016 - 2019), denominado "Barranquilla: Capital de Vida" en el que se fijaron objetivos, políticas, estrategias, programas y proyectos para la ejecución del mismo a través de su equipo de gobierno conformado por sus Secretarías Distritales (16 actualmente), oficinas, dependencias adscritas y demás entidades vinculadas a la administración distrital a través de contratos y/o convenios interadministrativos.

En materia de autonomía administrativa y financiera, el Distrito de Barranquilla, contempló en el artículo 74 de su plan de desarrollo 2016 - 2019, el "Programa Fortaleciendo La Alcaldía" como aquel "que busca mejorar el desempeño de los procesos para brindar productos y servicios con calidad a la comunidad", (Acuerdo Distrital No. 00112016 "POR EL CUAL SE ADOPTA EL PLAN DE DESARROLLO DISTRITAL, 2016 -2019) en el cual, se encuentra asociado el Proyecto de "Sistemas de Administración de Bienes y Servicios Públicos", que contempla el debido ingreso y registro de los activos distritales a los estados financieros y en el banco de proyectos y asignado su código BPIN. En lo que va del período de gobierno actual, hasta la vigencia de 2017. A través de la gestión mencionada y desarrollada por la Secretaría General del Distrito, se ha alcanzado un alto grado de eficacia, en cuanto a lo que refiere a la administración de los activos distritales a través de la actualización del 
Banco de Tierras de los bienes muebles e inmuebles de propiedad del Distrito de Barranquilla, pues con esta labor, se han optimizado recursos e ingresos y se han reducido costos de inversión en el desarrollo de obras de infraestructura, como por ejemplo en el caso reciente de intervención, adecuación y remodelación de los escenarios deportivos, en virtud al compromiso social, cultural y deportivo de celebrar los pasados Juegos Centroamericanos y del Caribe 2018, del cual Barranquilla fue epicentro, cumpliendo con el compromiso establecido en el artículo 16 de su plan de desarrollo "Barranquilla: Capital de vida" de "convertir este gran evento en una oportunidad para la transformación urbana de Barranquilla" (Acuerdo Distrital No. 0011 2016 "POR EL CUAL SE ADOPTA EL PLAN DE DESARROLLO DISTRITAL“, 2016 2019 ) Gracias al saneamiento de titularidad y legalización de bienes inmuebles de propiedad del Distrito de Barranquilla, se redujeron costos de inversión destinados a compra de inmuebles para el funcionamiento de estos escenarios, como en el caso la Pista BMX, Pista De Atletismo - Complejo Deportivo Pibe Valderrama, Softball, Piscina Olímpica, Coliseo Sugar Baby, antiguo Coliseo Humberto Perea.

Adicionalmente, es válido mencionar las optimizaciones de costos de intervención por parte de la administración distrital actual, en aquellos proyectos de prioridad legal, relacionados con el campo de la salud y educación. En esta parte, se destacan los proyectos de ampliación y remodelación de Hospitales, PASOS y CAMINOS, al igual de las infraestructuras para Instituciones Educativas Distritales (IED).

Es válido destacar que para la aprobación de un plan de desarrollo, sea este de orden nacional o territorial, es necesario vincular la participación de la ciudadanía y organizaciones civiles en la elaboración del mismo. En cumplimiento de esto, se están desarrollando programas de "Espacios para el Deporte y la Recreación", los cuales al ser ejecutados con la intervención, ampliación, adecuación y mantenimiento de predios de propiedad del Distrito y de aquellos legalizados a favor de este, a través de cesiones gratuitas con el apoyo de otras entidades públicas y declaraciones fiscales de bienes baldíos, no solo han generado un aporte social a la comunidad sino que además, han propiciado un aumento de resultados en programas relacionados con salud y educación, pues la cultura y la educación deportiva y recreativa coadyuvan con estos 
aspectos fundamentales. Ejemplo de esto, son las zonas verdes, canchas deportivas intervenidas en los diferentes barrios de la ciudad de Barranquilla.

Además de lo anterior, es procedente resaltar la Recuperación de activos distritales a través de procesos de restituciones fiscales, la captación de otros ingresos propios y disminución de detrimentos patrimoniales, realizados a través de procesos de baja de bienes, venta de inmuebles en proindiviso que no se encuentran en uso y no son requeridos como necesidad para la ejecución de diversos proyectos, así como la captación de ingresos nuevos a través de contratos de arriendos de aquellos predios que no se encontraban reportados en el banco de tierras como activos distritales y que a su vez, estaban siendo usados y explotados económicamente por particulares. Todo lo anterior, con previa autorización del Concejo Distrital a través de acuerdos sancionados a iniciativas del gobierno distrital actual.

De acuerdo a la legalización de estos activos, se ha logrado reportar al departamento de contabilidad como activo distrital al corte del año 2017, 746 inmuebles aproximadamente, con un avalúo catastral de \$99.259.318.459.00 M/L.

En atención a la captación de nuevos ingresos, se ha logrado un total de $\$ 2.940 .150 .222 .00 . \mathrm{M} / \mathrm{L}$.

Sin duda, el Distrito de Barranquilla con esta labor de administrar, legalizar, sanear y recuperar bienes muebles e inmuebles que se encontraban en uso propio y/o en explotación por parte de particulares, ha logrado aumentar sus activos, reducir sus costos de inversión frente al gasto público y ha mejorado mínimamente la sostenibilidad de sus proyectos. No obstante, y pese a los grandes resultados, aun se evidencian debilidades fuertes relacionadas con la administración y actualización del Banco de Tierras la cual a la fecha no ha logrado un $50 \%$ de ejecución, no se ha cumplido a cabalidad con los compromisos contables adquiridos para lo que va corrido del año 2018 , no se ha puesto un freno fuerte y severo a las invasiones ni se ha establecido un límite a la explotación de bienes del Distrito por parte de particulares, se sigue incidiendo en el déficit económico celebrando contratos de arriendos con particulares para el funcionamiento de Instituciones Educativas de orden Distrital, así como de 
oficinas y/o dependencias del mismo orden; la presunta manipulación contractual en proyectos de "bajo costo" con adiciones posteriores para ocultar el favorecimiento de algunos con la dedocracia de contratos, evitando someterlos a concursos y licitaciones públicas, de conformidad a lo dispuesto por el decreto 1082 de 2015 y la nueva Ley de contratación Pública No. 1882 de 2018; la demora de ejecuciones y entregas de obras públicas logrando con este adiciones que incrementan costos en las mismas, la falta de procedimientos y mecanismos de sostenibilidad de proyectos, entre otras.

\section{Conclusiones}

A la postre nos permitimos concluir el presente "paper" exponiendo algunas consideraciones que consideramos de pertinencia, así pues, la descentralización Fiscal, trae consigo un sinnúmero de debilidades Institucionales, en la medida en que se han ido agudizando los problemas fiscales y presupuestarios de los Estados, y han ido tomando impulso ciertas iniciativas en contra de las cargas tributarias, olvidando que el fundamento de estas, obedece a los principios de equidad e igualdad.

La organización territorial de nuestro país, en el modelo de descentralización no está alcanzando adecuadamente los resultados previstos, pues los principales problemas en materia fiscal son la deficiente gestión de las administraciones, asociado a prácticas de corrupción y falta de regulación; por lo tanto es menester que el gobierno nacional lidere una estrategia para garantizar que la descentralización cumpla con sus objetivos. Lo cual implicaría un reforzamiento de todos los instrumentos de seguimiento y control con énfasis en el impacto de los sectores principales, y de esta forma ofreciendo herramientas eficaces para el control fiscal de las finanzas territoriales.

La Descentralización en el Distrito de Barranquilla permitió la adopción de un nuevo régimen Político, Administrativo y fiscal logrando con esto la asignación de mayores recursos y beneficios para la ciudad de Barranquilla los cuales no se han aprovechado 
con mayor eficiencia por las administraciones deficientes y por la falta de seguimiento del gobierno para que se cumplan los objetivos previstos.

Si bien es cierto el distrito especial y portuario de Barranquilla haciendo uso de las atribuciones que como ente descentralizado le corresponden a logrado aumentar sus activos y reducir sus costos de inversión también lo es que se encuentra muy débil frente a las políticas de sostenibilidad fiscal teniendo en cuenta que sus fuentes captación y optimización de recurso propios carecen de vigilancia y control por lo tanto seria de suma importancia incrementar políticas de auto sostenimiento en cada proyecto y obra ejecutada de manera que estas permanezcan a largo plazo y así la ejecución de cada proyecto represente una verdadera inversión de aporte social a la comunidad y no un gasto publico de detrimento patrimonial o déficit fiscal.

\section{Referencias}

Acuerdo No. 006 de 2006 "Por el cual se Formaliza el proceso de descentralización interterritorial del Distrito de Barranquilla".

Acuerdo Distrital No. 00112016 "Por El Cual Se Adopta El Plan De Desarrollo Distrital ( "Barranquilla: Capital De Vida" - Artículo 742016 -2019).

Acuerdo Distrital No. 00112016 "Por El Cual Se Adopta El Plan De Desarrollo Distrital" (Barranquilla: Capital De Vida" 2016 -2019).

Constitución Política, Artículo 328 y 332 (1991).

Gonzalo Zapata, Juan (2008). Las finanzas territoriales en Colombia. Revista Virtual https://www.caf.com/media/3782/FinanzasTerritoriales.pdf 
Hernández Gamarra , A., \& Barreto Nieto, L. H. (Marzo de 2018). Descentralización y Finanzas Territoriales Cuadernos Fedesarrollo No. 59. Obtenido de https://www.fedesarrollo.org.co/sites/default/files/05reportedescentralizacion.pdf

Kalmanovitz, S. (1989). La encrucijada de la sinrazón y otros ensayos. En S. Kalmanovitz. Bogotá: Tercer Mundo.

Ley 60 de (1993). "Por la cual se dictan normas orgánicas sobre la distribución de competencias de conformidad con los artículos 151 y 288 de la Constitución Política se dictan otras disposiciones".

Ley 152 de (1994). "Por la cual se establece la Ley Orgánica del Plan de Desarrollo"

Maldonado, A. (2010). Los municipios de la "Otra Colombia" en la política de descentralización. Balance de la situación y propuestas. Obtenido de http://foros.uexternado.edu.co/ecoinstitucional/index.php/opera/article/view/123

Villa, G. A. (Junio de 2005). Ensayo“Colombia: Gasto Público Social Regional 1997 2003. Obtenido de Banco de la Republica I Colombia : www.banrep.gov.co/es/eser-26

Villanueva, F. R. (2011). PERSPECTIVAS FISCALES DEL DISTRITO. Revista Panorama Económico. Número 19, 113-134. 